\title{
SELECTIVE X-RAY BRAGG SPECTROMETRY: OPTIMIZING FLUORESCENCE MICROPROBE SENSITIVITY FOR PRECIOUS METALS
}

\author{
Barbara Etschmann ${ }^{1}$, Chris Ryan ${ }^{2}$, Stefan Vogt $^{3}$, Jörg Maser $^{3}$, Cathy Harland ${ }^{4} \&$ \\ Joël Brugger 5,6
}

${ }^{1}$ CSIRO Exploration \& Mining, Mineralogy, South Australian Museum, North Terrace, Adelaide, SA 5000, Australia ${ }^{2}$ CSIRO Exploration \& Mining, School of Geosciences, Building 28E, Monash University, Clayton, VIC 3168, Australia

${ }^{3}$ Experimental Facilities Division, Advanced Photon Source, Argonne National Laboratory, 9700 S. Cass Ave, Argonne IL 60439 USA

${ }^{4}$ Australian Synchrotron Research Program (ANSTO), Advanced Photon Source, Argonne National Laboratory, 9700 S. Cass Ave, Argonne IL 60439 USA

${ }^{5}$ Mineralogy, South Australian Museum, North Terrace, Adelaide, SA 5000, Australia

${ }^{6}$ CRC LEME, School of Earth and Environmental Sciences, University of Adelaide, Adelaide SA 5005, Australia

\section{INTRODUCTION}

The knowledge of the speciation of trace metals at micro- to nano-scale in soil and sediments is fundamental to understanding and predicting element mobility, bioavailability and biotoxicity. X-ray microprobes have seen much improvement in last decade due to the advent of third generation synchrotron sources and progress in focussing mirror and detector technology. Some of the advantages of this technique are:

- Sub-micron resolution;

- Low detection limits (ca. 1 ppm);

- Non-destructiveanalysis (at least for inorganic samples);

- Ability to study wet samples or samples under controlled environment;

- Ability to combine concentration information (x-ray fluorescence) with speciation information ( $\mathrm{x}$ ray absorption near-edge spectroscopy).

The next challenge is in rendering the instrument more user-friendly and in reducing detection limits. We present here the results from an instrument development project aimed at developing an X-ray spectrometer configuration for the X-ray fluorescence microprobe optimized for the detection of precious metals in geological and biological samples. The objective is to improve detection limits and reduce interference from major elements and beam scatter. The approach used Bragg diffraction from a surface shaped to form a segment of logarithmic spiral (log-spiral) to focus X-rays from precious metals onto a solid-state detector. The result is an enhancement of precious metal lines within the narrow band-pass of the Bragg crystal surface with suppression of detector artefacts such as tailing and escape peaks arising from interfering elements and scattered photons.

\section{BACKGROUND}

Gold in ore can be economic at concentrations of a few ppm, depending on ore mineralogy and the nature of gold residence in the ore. Consequently, interest in the distribution of gold between coexisting sulphide minerals in ore demands the imaging and analysis of gold at ppm to sub-ppm levels. Similarly, thermodynamic data suggest that the concentrations of gold in the fluids involved in ore formation in magmatic hydrothermal systems are seldom higher than a few ppm. Hence, the study of ore fluids trapped and preserved as fluid inclusions in minerals necessitates the detection of gold at ppm to sub-ppm levels during in situ analysis of fluid inclusions. Gold anomalies in soils and calcrete are often in the range of $10 \mathrm{~s}$ to 100s of ppb; knowledge of gold speciation in these fine-grained samples is fundamental for understanding the origin of these anomalies and in ranking anomalies to select the most prospective exploration targets.

In the biomedical sciences, platinum in the form of cis-platin plays a major role in many chemotherapeutic drugs. Mapping and quantifying platinum distributions within tissues and single cells is therefore of great interest, e.g., to study the mode of action of chemotherapy drugs on tissues and cells on a sub-cellular level, as well as how these are altered in cells that develop resistivity against a specific drug. This knowledge could then be applied to the development and testing of new chemotherapeutic agents.

However, the low concentration of precious metals, such as Au and Pt, in geological and biological samples often challenges the detection sensitivity of high-resolution microanalytical probes. Destructive probes such as laser ablation can provide sensitive point analysis of geological material at low spatial resolution $(\geq 100$ $\mu \mathrm{m}^{2}$ ), but not detailed spatial images of concentration. SIMS can provide spatial data at high sensitivity with 
some resolution compromise, but is difficult to quantify. Neither technique is suited to delicate biological samples. Synchrotron X-Ray Fluorescence (SXRF), using a focused keV photon beam, and Proton Induced $\mathrm{X}$-ray Emission (PIXE), using a focused MeV proton beam, provide non-destructive analysis based on well characterized X-ray production mechanisms that lend themselves to standardless quantitative analysis, and real-time imaging capabilities with micron spatial resolution. Furthermore, these techniques permit the analysis and imaging of buried structures, such as fluid inclusions and microscopic precious metal minerals or individual cells in tissue or cultures. However, the concentration of precious metals often fall tantalizingly below the detection limits of these techniques using conventional solid-state detection approaches.

A distinguishing feature of SXRF is the use of selective excitation of elements of interest, allowing the suppression of an interfering major or minor element. A major analytical interference for Au detection using $\mathrm{L}$ X-ray lines is the common association of $\mathrm{Au}$ with As in sulphide geological samples. However, the As $\mathrm{K}$ edge is lower in energy than any Au L edge, which eliminates this approach for enhancing gold detection; any beam energy that excites a Au L sub-shell will also excite As. Similarly, photon beam energies chosen to optimize the detection sensitivity of Pt in biological samples cause tailing in solid-state detectors, arising from incomplete charge collection, that produces an elevated background under the Pt L lines which degrades ultimate detection sensitivity. Increasing the energy separation between Pt lines and beam energy in order to reduce tailing also reduces the ionisation cross-section nullifying any potential gain.

Johansson double focussing crystal spectrometers based on Bragg diffraction offer high-energy resolution, which enable precious metal lines to be resolved from interferences. However, these spectrometers have low detection solid angles, which leads to low counting rates making them unsuitable for imaging applications. Similarly, high-resolution spectrometers based on microcalorimetry techniques have great potential for spectroscopy but lack high solid angles.

Advances in Bragg diffraction materials offer great potential to remedy this situation by permitting spectrometer designs combining selectivity with large effective solid angles. Techniques have been demonstrated for the fabrication of films of Highly Ordered Pyrolytic Graphite (HOPG) on surfaces of virtually arbitrary shape (Grigorieva et al. 2003). These techniques permit construction of surfaces of $<002>$ oriented HOPG with radii of curvature as small as $5 \mathrm{~mm}$. Hence, surfaces can be designed for optical properties rather than for ease of fabrication. For example, the method has been applied to the construction of toroidal energy band-pass filters (Kolmogorov \& Trounova 2003).

In 1914, de Broglie \& Lindermann noted that the surface that provides an equal Bragg diffraction angle for all rays radiating from a point source follows a log-spiral curve (de Broglie \& Lindemann 1914). The new HOPG techniques permit these surfaces to be fabricated and coated with ca. $200 \mathrm{~mm}$ of $<002>$ oriented graphite. The broad mosaic spread of these films (ca. $0.4^{\circ}$ ) also contributes to high peak reflection efficiencies (integrated reflectivity of ca. $0.2^{\circ}$ ) (Grigorieva et al. 2003).

A recent approach using HOPG coated surfaces based on a log-spiral led to strong dispersion in the analysed beam with diffracted photons detected using a p-i-n-diode array (Grigorieva et al. 2003, Pease et al. 2000), similar to log-spiral bent Laue crystal spectrometers. The present work utilizes an alternate geometry designed at the CSIRO that combines a log spiral surface of rotation with a focussing action similar to a toroidal analyser in order to concentrate the selected photons into a solid-state detector (Figure 1). The energy-dispersive solid-state detector will provide further discrimination of interferences arising from scattered photons. The focussing effect leads to a large effective solid angle for photons satisfying the Bragg condition. The result is a simple Bragg Filter accessory for a solid-state detector, which combines rejection of unwanted lines and detector artefacts with efficient collection of signal from precious metals. This facilitates the direct imaging of precious metal distribution to low detection levels with interferences minimised. The current system is described below.

\section{THE EXPERIMENT}

X-ray fluorescence data were collected at the 2ID-E microprobe at the APS, using an incident

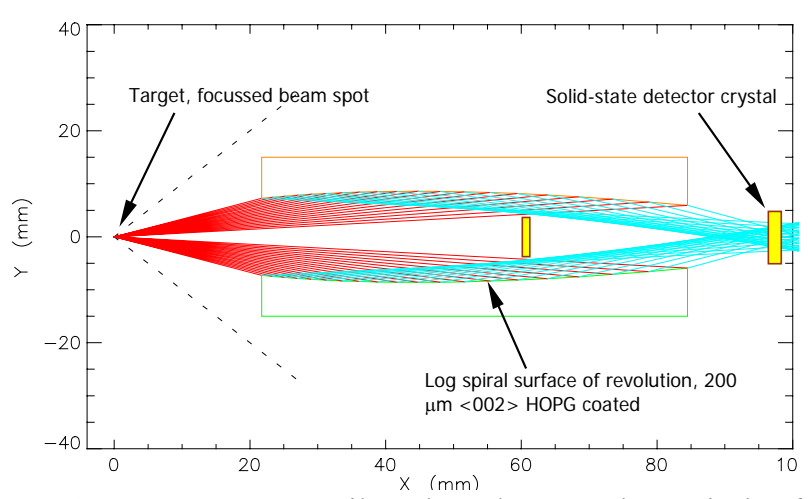

Figure 1: Bragg Filter based on a log-spiral of evolution. 
beam energy of $12.1 \mathrm{keV}$. 2-ID-E is a branch beam-line that uses a silicon crystal splitter as a monochromator. For these experiments, $20 \mathrm{~cm}$ zone plates were used, resulting in a focused beam to a spot of 1.1-1.5 microns. An order-sorting aperture intercepted higher diffraction orders and most of the transmitted beam. A single element Ge energy-dispersive detector was located at ca. 90 degrees to minimize detection of the scattered beam and fitted with a conical collimator large enough to accept scattered X-rays from the Bragg Filter.

The Bragg Filter, located between the sample and the detector, was placed on a motorized stage to allow the filter-sample distance to be optimized for a particular energy. The alignment of the filter was tuned by scanning the position of the filter (lateral and distance from the sample) to align the focus of the log-spiral cylinder on the focused beam spot on the sample. The filter-to-sample distance was optimized by checking the intensity of the Au signal from a standard VFeAu foil (ratio of elements 13:7:80) and a pyrite sample from the Emperor Mine, Fiji that had been well characterized using both PIXE and SXRF.

100-500 second line scans were collected on the NIST 1832/1833 standard foils, a NIST 610 standard glass, a polished sample of pyrite from the ore zone of the Emperor Mine, Fiji, and a clay containing almost $1 \mathrm{ppm}$ $\mathrm{Au}$, with and without the filter.

Imaging was done by raster scanning the sample through the focused beam. Full spectra were acquired at each scan position, thus there was a minimum collection time of 0.5 second per pixel. The clay sample was imaged with (ca. $7 \mathrm{~h}$ ) and without (ca. $2 \mathrm{~h}$ ) the filter. The Fiji pyrite sample was only imaged with the filter (ca. $11 \mathrm{~h}$ ), given that it had already been imaged previously (ca. $7 \mathrm{~h}$ at $16.1 \mathrm{keV}$ ), without a filter.

Preliminary results, based on the line scans from the Fiji pyrite sample, demonstrate the filter response function (Figure 2). The ratio of the peak areas using the filter to not using the filter shows an increase of ca. 4 at $\mathrm{E}$ ca. $9.7 \mathrm{keV}$. The Au L $\alpha 1$ peak area was increased by a factor of 3.89. The As K $\alpha 1$ peak area was also increased, but only by a factor of 1.18. Lines below an energy of $8.6 \mathrm{keV}(\mathrm{Zn}$ $\mathrm{K} \alpha 1)$ were suppressed by an order of magnitude, while lines with an energy greater than $11.5 \mathrm{keV}$ (Au L $\beta 2$, As K $\beta 1$ ) were suppressed by a factor of 5 . The Rayleigh beam scatter peak, which is visible as a small tail on the As $\mathrm{K} \beta 1$ peak in Figure 3a, was suppressed on the spectrum collected with the filter (Figure $3 \mathrm{~b}$ and $3 \mathrm{c}$ ).

This filter response function was fitted with a Gaussian giving the fitting parameters listed in Figure 2.

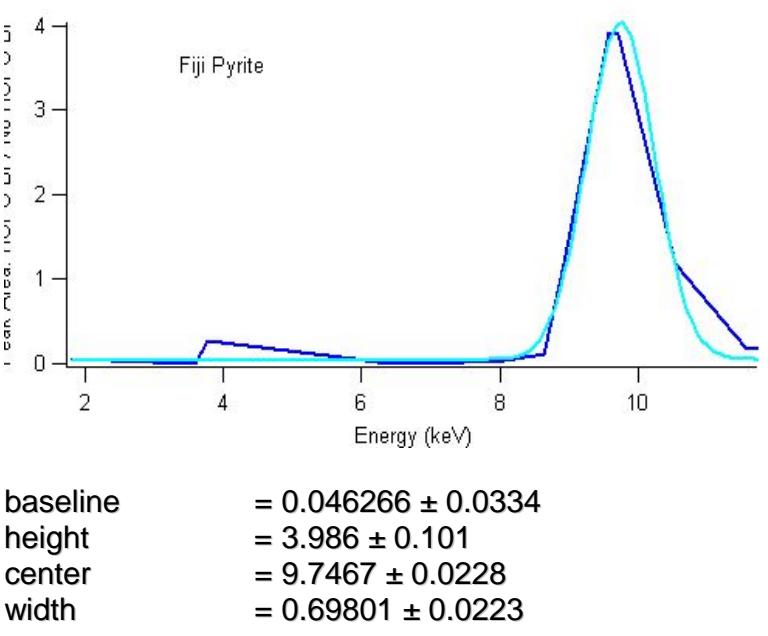

Figure 2: HOPG Bragg Filter response function.

X-ray relative intensities calculated by GeoPIXE II (GeoPIXE software; Ryan 2000) were multiplied by the Gaussian (with width adjusted to 0.5) in order to fit the spectra. The resulting fit approximates the experimental spectrum quite well (Figure 3c). It should be noted that the calculated peak between $\mathrm{Cu} \mathrm{K} \alpha 1$ and $\mathrm{Cu} \mathrm{K} \beta 1$ (due to $\mathrm{Au} \mathrm{Ll}+\mathrm{Zn} \mathrm{K \alpha 1}$ ) is too large and this still needs to be accounted for.

The Fiji pyrite $\mathrm{Au}(\mathrm{L})$ images collected with $(12.1 \mathrm{keV})$ and without $(16.1 \mathrm{keV})$ the filter are compared in Figure 4.

The observed zonation using the Bragg Filter appears to show better definition. Not only do the trace-level $\mathrm{Au}$ zones appear more detailed in the image that was collected with the filter, an extra zone can be seen, highlighted by the green box.

The Gaussian response function was applied to fit the clay spectra (Figure 5). A first-order approach to deal with the relatively sharp rise in the higher energy end of the spectrum was to change the baseline from a constant to a simple linear function $(\mathrm{y}=100.0 * \mathrm{E}+0.046)$. While this improved the fit, more work still needs to be done to properly account for effects of the Bragg Filter response on the continuum spectrum. Unfortunately, peaks due to Au still remain undetectable in either spectrum. 

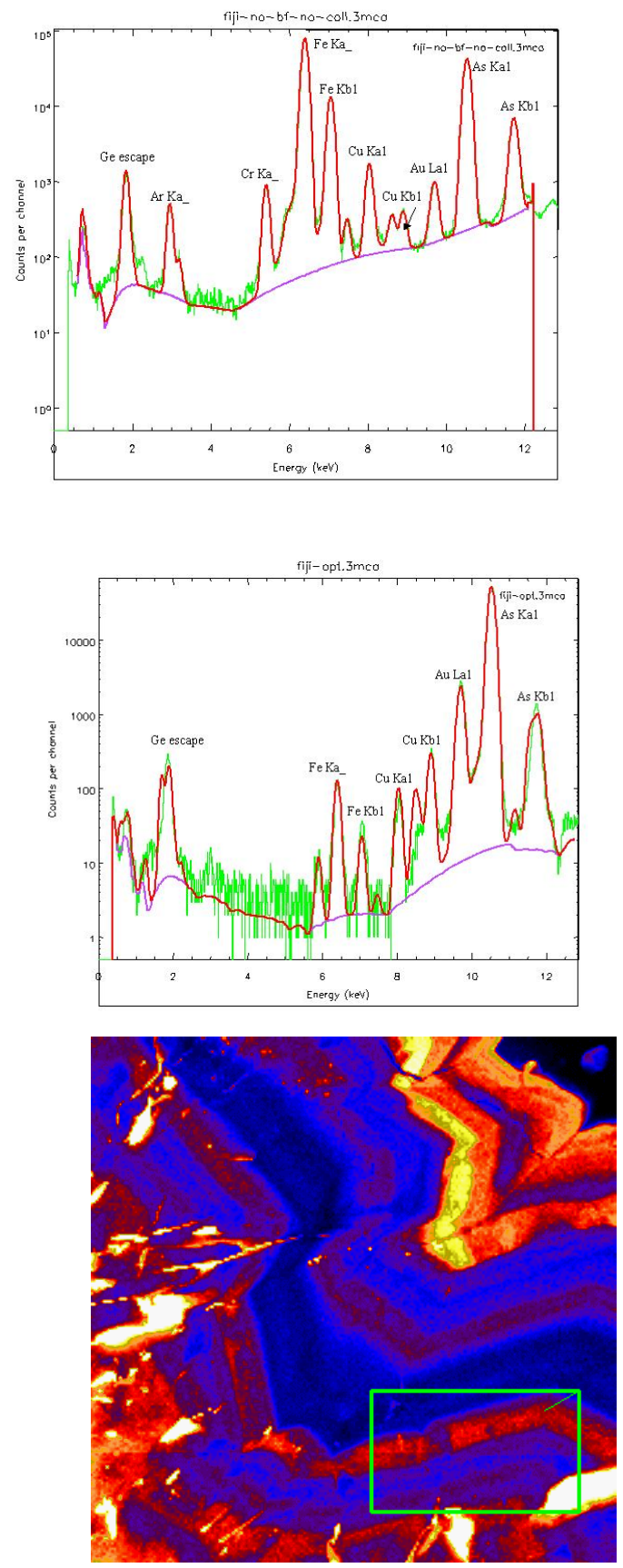

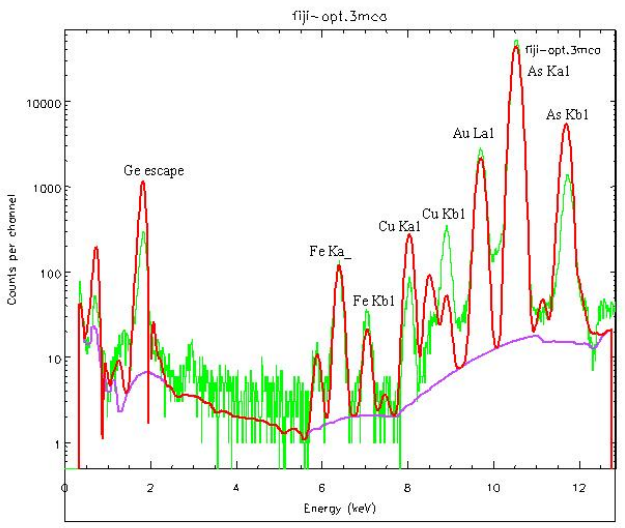

Figure 3a (top left): is the Fiji Pyrite spectrum collected without HOPG Bragg Filter. Using "standard" $\mathrm{X}$-ray relative intensities to fit the spectrum.

Figure 3b (top right): is the Fiji Pyrite spectrum collected with HOPG Bragg Filter. Using "standard" Xray relative intensities to fit the spectrum.

Figure 3c (bottom left): is the Fiji Pyrite spectrum collected with HOPG Bragg Filter. Using "standard" Xray relative intensities multiplied by the Gaussian filter response function to fit the spectrum.

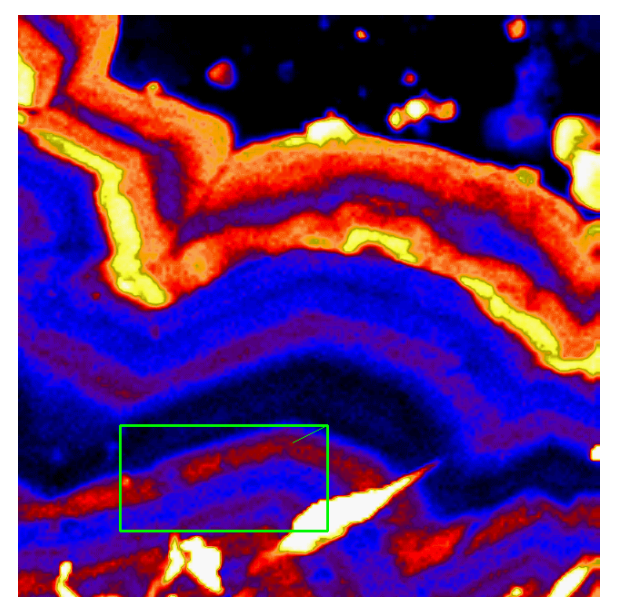

Figure 4: Image of $\mathrm{Au}(\mathrm{L})$ in Fiji Pyrite sample. Left: Imaged at $12.1 \mathrm{keV}$ with the Bragg Filter in optimised position. Right: Imaged at $16.1 \mathrm{keV}$ without the Bragg Filter. Maximum displayed concentrations of elements are: $\mathrm{Fe} \sim 45.4 \mathrm{wt} \%, \mathrm{Cu} \sim 0.8 \mathrm{wt} \%$, As $\sim 3.5 \mathrm{wt} \%$ and $\mathrm{Au} \sim 0.4 \mathrm{wt} \%$.

\section{CONCLUSIONS}

The HOPG Bragg Filter is relatively easy to use and align, and the use of the filter results in:

- Enhanced Au signal (factor of ca. 4), and enhanced detection limits

- Rejection of close interferences (As) together with their tail under the Au peak, further contributing to lower detection limits for $\mathrm{Au}$.

There are still some problems however. In particular, more work is required to properly account for effects of the Bragg Filter on the continuum spectrum. At the moment we are still unable to image Au at concentrations of less than 1 ppm. 
Acknowledgements: This work was supported by the Australian Synchrotron Research Program, which is funded by the Commonwealth of Australia under the Major National Research Facilities Program. Use of the Advanced Photon Source was supported by the U.S. Department of Energy, Office of Science, Basic Energy Sciences, under Contract No. W-31-109-Eng-38.
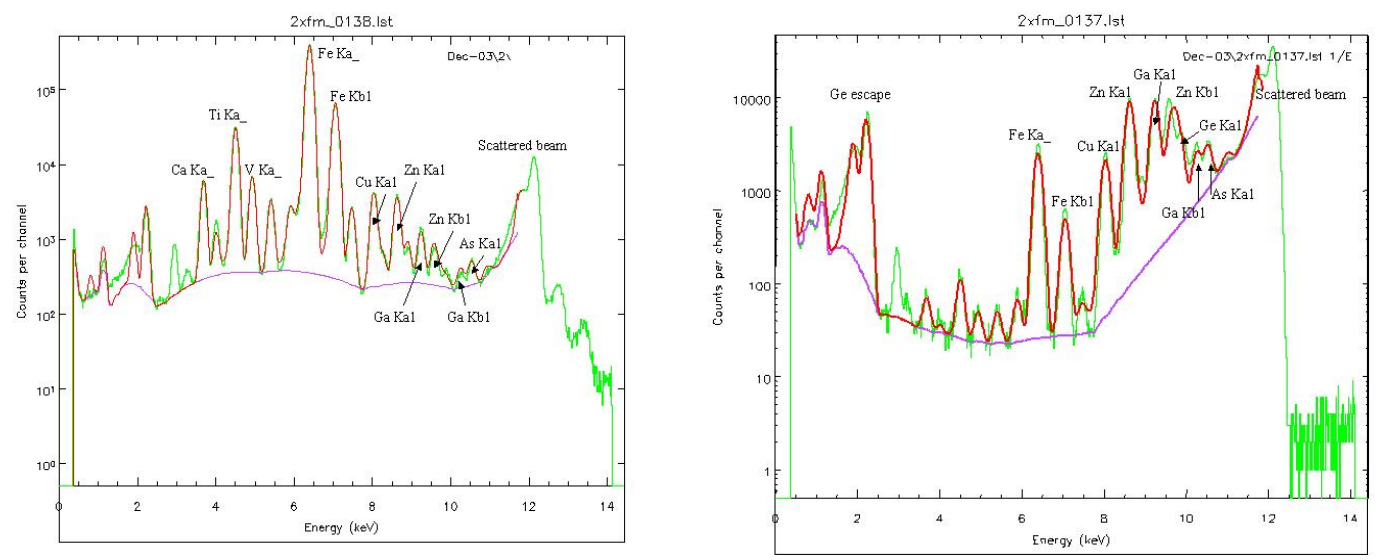

Figure 5: Spectra of the clay sample with almost $1 \mathrm{ppm}$ Au. Left is the clay spectrum collected without the HOPG Bragg Filter. "Standard" X-ray relative intensities were used to fit the spectrum. Right is the clay spectrum collected using HOPG Bragg Filter. "Standard" X-ray relative intensities multiplied with the Gaussian filter response function and extra background term were used to fit the spectrum.

\section{REFERENCES}

DE Broglie M. \& Lindemann F.A. 1914. Compt. Rend. 158, 944.

GeOPIXE SOFTWARE PACKAGE. http://nmp.csiro.au/GeoPIXE.html.

Grigorieva I.G. \& ANTONOV A.A. 2003. HOPG as powerful X-ray optics. X-ray Spectrometry 32, 64-68.

Kolmogorov Y. \& TROUNOVA V. 2003. Analytical potential of EDXRF using toroidal focussing systems of highly oriented pyrolytic graphite (HOPG). X-ray Spectrometry 31, 432-436.

PeAse D.M., DANIEL M. \& BUDNIK J.I. 2000. Log spiral of revolution highly oriented pyrolytic graphite monochromator for fluorescence x-ray absorption edge fine structure. Review Scientific Instrum. 71, 3267-3273.

RYAN C.G. 2000. Quantitative Trace Element Imaging using PIXE and the Nuclear Microprobe. International Journal of Imaging Systems and Technology, Special issue on "Advances in Quantitative Image Analysis” 11, 219-230. 